\title{
Erratum to: Affluent effluent: growing vegetables with wastewater in Melbourne, Australia - a wealthy but bone-dry city
}

\section{Fiona Barker-Reid • Grant A. Harper • Andrew J. Hamilton}

Published online: 24 March 2010

(C) Springer Science+Business Media B.V. 2010

\section{Erratum to: Irrig Drainage Syst DOI 10.1007/s10795-009-9082-x}

There were a few errors with the reporting of units in Table 1 (V and Enteric virus conc. in water) of the original paper, hence, these have been corrected in the table below.

Note that these were merely typographical errors and all calculations were performed using the correct units.

The results reported in the original manuscript are thus correct.

Table 1 Point estimates/probability distributions for exposure model parameters

\begin{tabular}{|c|c|c|}
\hline Parameter & Value/distribution & Source \\
\hline $\begin{array}{l}V=\text { vol. of water on plant's } \\
\text { surface }\left(\mathrm{mL} \mathrm{g}^{-1}\right)^{\mathrm{a}}\end{array}$ & Discrete uniform & (Hamilton et al. 2006) \\
\hline $\begin{array}{l}M=\text { mass of product consumed } \\
\quad \text { per day }\left(\mathrm{g} \mathrm{d}^{-1}\right)\end{array}$ & 21.81 & (ABS 1999, 2008b) \\
\hline $\begin{array}{l}k=\text { virus kinetic decay } \\
\text { constant }\left(\mathrm{d}^{-1}\right)\end{array}$ & Normal $(\mu=1.07, \sigma=0.07)$ & (Petterson et al. 2001a, 2002) \\
\hline $\begin{array}{l}\text { Enteric virus conc. in reclaimed } \\
\text { wastewater (commercial) } \\
\left.\text { (no. } \mathrm{mL}^{-1}\right)\end{array}$ & $9.99999 \times 10^{-7}$ & (EPA 2003) \\
\hline \multicolumn{3}{|l|}{$\begin{array}{l}\text { Thermotolerant or faecal } \\
\text { coliform conc. in greywater } \\
\text { (household) }\left(\mathrm{TC}^{\mathrm{b}} \mathrm{mL}^{-1}\right)\end{array}$} \\
\hline Laundry & Uniform $(0.09,160)$ & (Siegrist et al. 1976) \\
\hline Bathroom & Uniform $(0.01,33)$ & $\begin{array}{l}\text { (Christova-Boal et al. 1996; } \\
\text { Feachem et al. 1983; Siegrist et al. 1976) }\end{array}$ \\
\hline Kitchen & Uniform $(10,000,398,107)$ & $\begin{array}{l}\text { (Halalsheh et al. 2008; Naturvårdsverket } 1995 \\
\text { in Ottosen and Stenstrom 2003) }\end{array}$ \\
\hline Combined & $\begin{array}{l}(\text { laundry } \times 0.44)+(\text { bathroom } \times 0.38)+ \\
(\text { kitchen } \times 0.18)\end{array}$ & (ATA 2005) \\
\hline
\end{tabular}

${ }^{a}$ Water capture data for Winter Head cabbage was used to represent the worst case scenario as it captures more water on average than Savoy cabbage or broccoli

${ }^{\mathrm{b}} \mathrm{TC}=$ thermotolerant coliforms

The online version of the original article can be found under http://dx.doi.org/10.1007/s10795-009-9082-x.

F. Barker-Reid $(\bowtie) \cdot$ G. A. Harper $\cdot$ A. J. Hamilton

Department of Resource Management and Geography, Melbourne School of Land and Environment, The University of Melbourne, Melbourne, Victoria 3010, Australia

e-mail: s.barker-reid@pgrad.unimelb.edu.au 